\title{
Applications of velocity model building using Monte Carlo simulations in the Campos Basin, Brazil
}

Tiago Alcantara, Jon Burren, Tony Martin, Marcus Bell and Leonardo Pinheiro, PGS

\section{Copyright 2021, SBGf - Sociedade Brasileira de Geofísica}

This paper was prepared for presentation during the $17^{\text {th }}$ International Congress of the Brazilian Geophysical Society held in Rio de Janeiro, Brazil, 16-19 August 2021.

Contents of this paper were reviewed by the Technical Committee of the $17^{\text {th }}$ International Congress of the Brazilian Geophysical Society and do not necessarily represent any position of the SBGf, its officers or members. Electronic reproduction or storage of any part of this paper for commercial purposes without the written consent of the Brazilian Geophysical Society is prohibited.

\begin{abstract}
Velocity model building (VMB) is a time-consuming process involving several passes of migration and tomography. Martin and Bell (2019) proposed an automated stochastic approach that can accelerate the VMB turnaround. The method creates multiple random perturbations of the initial velocity model that are later inverted through tomography. The products of each individual perturbation are statistically combined and added to the starting model. This process is then repeated until a convergence criterion is met (flat gathers). In this abstract we will present an application of the Monte Carlo based VMB in the Campos Basin, Brazil. Additionally, we will demonstrate that the stochastic VMB produces velocity models, in accelerated turnaround, suitable for Full Waveform Inversion.
\end{abstract}

\section{Introduction}

Velocity models are essential for positioning and focusing seismic energy in the subsurface. The velocity estimation process is often convoluted and depends on various factors including data quality and geological complexity of the area. The process involves various loops of migration and tomography interleaved by quality controls within each loop (stop-go approach). To minimize human intervention, and reduce turnaround, Martin and Bell (2019) proposed an automated velocity model building approach using Monte Carlo simulations to efficiently converge to a global minimum.

In this paper we will present an application of the Monte Carlo based VMB demonstrating accurate and accelerated model building in the Campos Basin, Brazil. Additionally, we evaluate if this stochastic VMB approach produced a velocity model suitable to FWI.

\section{Method}

In order to perform a stochastic velocity model building both migration and tomographic engines must be efficient to ensure that a reasonable number of realizations can be performed in a short amount of time. Therefore, the workflow proposed by Martin and Bell (2019) combines Beam migration (Sherwood et al., 2008) and wavelet shift tomography (Sherwood et al., 2011) in a stochastic framework.

A schematic of the process is shown in Figure 1. The automated process starts with a migration using an initial velocity model. A population of random perturbations are then created and added to the starting model. All the velocity models are then updated through tomography and, later all the velocity differences are statistically combined and added to the starting model. The initial model is therefore modified and, as the automation continues, the model building evolves. The process is repeated several times, with measures of convergence being generated after each cycle (gather flatness). Once the convergence criterion of gather flatness is met globally, the process stops, and a usable velocity model is generated.

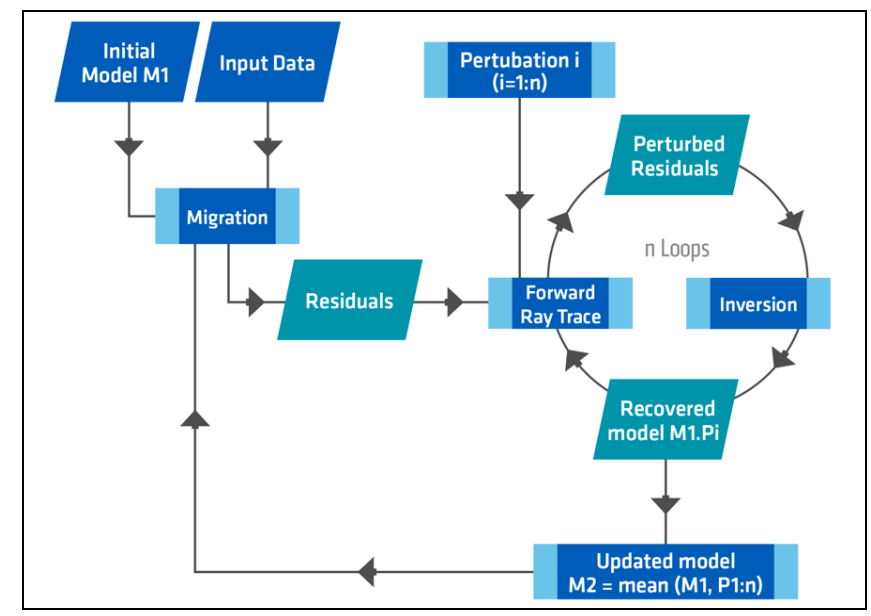

Figure 1. Stochastic VMB workflow.

Martin et al. (2020) demonstrated the benefits for both quality and time using Monte Carlo simulation for VMB on a West Africa dataset. In that exercise, they also showed that the automated VMB achieved comparable results to the conventional 'stop-go' approach. Here we will use the automated VMB to estimate a velocity model for an early look Reverse Time Migration (RTM); additionally, we evaluate if the estimated model is suitable for Full Waveform Inversion.

\section{Results: velocity model building}

An attempt to obtain a velocity model for an early look RTM was performed using a recently acquired survey in Campos Basin, Brazil. This was a multisensor streamer acquisition with 14 cables, $100 \mathrm{~m}$ separation and $10 \mathrm{~km}$ maximum offset covering over $5000 \mathrm{~km}^{2}$.

The initial velocity model was created in a two-step process; building the water velocity profile and the sediment trend. The water velocity profile was created using temperature-salinity measurements (TS-dip) and the sediment trend was a simple gradient tied to the water bottom horizon, $\mathrm{V}_{0}+\mathrm{KZ}$, where $\mathrm{V}_{0}=1600 \mathrm{~m} / \mathrm{s}, \mathrm{K}=0.85$ and $Z$ is the depth in meters. 
The VMB took less than a month and consisted of three main stages: 1) post carbonate/salt velocity updates using Monte Carlo simulation; 2) carbonate and salt interpretation, and 3) presalt velocity update using the automated VMB approach. The post-salt updates consisted of 6 cycles of the automated approach using 20 perturbations and a $30^{\circ}$ mute applied to the gathers. Carbonates and salt were inserted to the model, and the Monte Carlo simulation then solved the presalt section. The velocity update in the presalt consisted of 3 cycles of the pseudo-random approach with 20 perturbations and a $25^{0}$ mute.

Figure 2 shows gathers and velocity model before (top) and after (bottom) the full accelerated VMB. The initial common image gathers (CIGs) present significant underand over-correction. After the velocity updates, gather flatness improves and, consequentially, better focusing and positioning of events in the stack can be noticed (Figure 3).

The automated flow provided a velocity model for an early RTM volume that allowed a preliminary analysis of the area and improved planning for future work: a detailed velocity model building and depth migration, which includes FWI and a Least Squares Migration (LSM).

\section{Results: Full Waveform Inversion}

Once the velocity model was estimated, an additional exercise was performed to evaluate if the stochastic VMB approach produced a velocity model appropriate for FWI. A robust starting velocity model is crucial to the inversion of seismic reflection data through $\mathrm{FWI}$ techniques since it minimizes the risk of cycle skipping.

FWI estimates a velocity field by minimizing the difference between modeled and observed data. In this work both refractions and reflections were used to update the earth model. The modeled data was created using the vectorreflectivity method described by Whitmore et al. (2020). This approach does not require a density function to produce reflections. Instead, a reflectivity volume allows reflections to be modeled, that can be used by $\mathrm{FWI}$ in updating the velocity model. In addition to this, we used an inverse scattering-based gradient to update the low wavenumber component of the velocity model, without interference from the migration isochrones (RamosMartinez et al., 2016).

Initially, a modeling exercise was performed to evaluate the suitability of the model for FWI, by comparing synthetic and observed data, in particular look at the potential for any cycle skipping. A quantitative analysis was performed by analyzing a cross-plot of the crosscorrelation coefficient and the time lag (Figure 4). Each point on the cross-plot represents measurements for a shot gather. After successful updates using FWI, the cross-correlation coefficient will converge towards one and, the time lag will tend to zero. To successfully update the velocity field using a $10 \mathrm{~Hz} F W I$, the time lag between the two datasets must be within $\pm 50 \mathrm{~ms}$. According to the analysis, the Monte Carlo based approach produced a velocity model with a time lag range within $\pm 40 \mathrm{~ms}$ and, therefore, suitable to FWI (top image in Figure 4).
After evaluating the time lag between synthetic and observed datasets, a $10 \mathrm{~Hz} F W I$ was performed using offsets from $0.5 \mathrm{~km}$ to $10 \mathrm{~km}$, targeting improvements in the post salt sediments and ideally refinement of the top of salt. The input data was muted using the top of salt mask created during the velocity model building. Twenty iterations of FWI were performed using the non-specular kernel described by Ramos-Martinez et al. (2016).

The quality control of the FWI was divided into two parts: data-domain and image-domain. In the data-domain QC we evaluated the improvements in match between modeled and observed data. In this work we assess how the match between these two datasets improved by evaluating a cross-plot of the cross-correlation coefficient and the time lag (Figure 4). As expected, the match of the two datasets has considerably improved after 20 iterations of $\mathrm{FWI}$, and the cross-plot shows a tight clustering focused close to a cross-correlation coefficient of one and a time lag of zero (bottom image in Figure 4).

Image-domain QC was performed by analyzing gather flatness, velocity models, velocity perturbation, and stack response. In Figure 5, we present the common image gathers using the input model to FWI (top) and after the inversion (bottom). As shown, FWI improved gather flatness. Later, the velocity models were also evaluated to ensure that the velocity update was smooth no artifacts were introduced. Figure 6 shows the velocity models before (top) and after the inversion (middle) and, as expected, the velocity update is smooth without migration isochron artifacts (bottom image in Figure 6). Finally, Figure 7 shows the stacks before (top) and after (bottom) the inversion. The updates provided by FWI have reduced velocity errors in the subsurface, improving focusing and positioning at the top salt (white arrow).

According to results presented, the stochastic VMB approach produced a velocity model, in a reduced turnaround, suitable for FWI. As the velocity model building would progress, additional FWI and ray-based tomographic passes would be necessary to further refine the earth model, thus improving gather flatness and, consequentially, the subsurface image.

\section{Conclusions}

Velocity models are crucial in producing reliable images of the subsurface. Velocity model building can be cumbersome and depends on various factors including data quality and the geological complexity of the area. The process involves various loops of migration and inversion interleaved by quality controls within each loop. To minimize human intervention, and accelerate turnaround, Martin and Bell (2019) proposed an automated velocity model building approach that uses Monte Carlo simulations to efficiently converge to a global minimum.

In this work we presented an application of the stochastic velocity model building. We used a recently acquired Campos Basin dataset to produce a velocity model for an early look RTM. Additionally, we also demonstrated that the velocity model provided by the Monte Carlo based approach is suitable for FWI. 


\section{Acknowledgments}

We thank PGS for permission to publish the results.

\section{References}

Martin, T., and Bell, M., [2019]. An innovative approach to automation for velocity model building: First Break, 37, no. 6, 57-66.

Martin, T., Bell, M., and Massip, T., [2020]. Automating velocity model building using Monte Carlo simulations - A West African case study, EAGE2020 Annual Conference Online.

Ramos-Martinez, J., Crawley, S., Zou, K., Valenciano, A.A., Qiu L., and Chemingui N., [2016]. A robust gradient for long wavelength FWI updates, 78th EAGE Conference \& Exhibition, Extended Abstracts.

Sherwood, J.W.C., Sherwood, K., Tieman, H., and Schleicher, K., [2008]. 3D beam prestack depth migration with examples from around the world. 78th SEG Annual International Meeting, Expanded Abstracts, 438-442.

Sherwood, J., Jiao, J., Tieman, H., Sherwood, Zhou, K., Lin, C. S., and Brandsberg-Dahl, S., [2011]. Hybrid tomography based on beam migration: 81st Annual International Meeting, SEG, Expanded Abstracts, 3979-3983.

Whitmore, N.D., Ramos-Martinez, J., Yang, Y., and Valenciano, A.A., [2020]. Full wavefield modeling with vector-reflectivity, 82nd EAGE Conference \& Exhibition, Extended Abstracts.
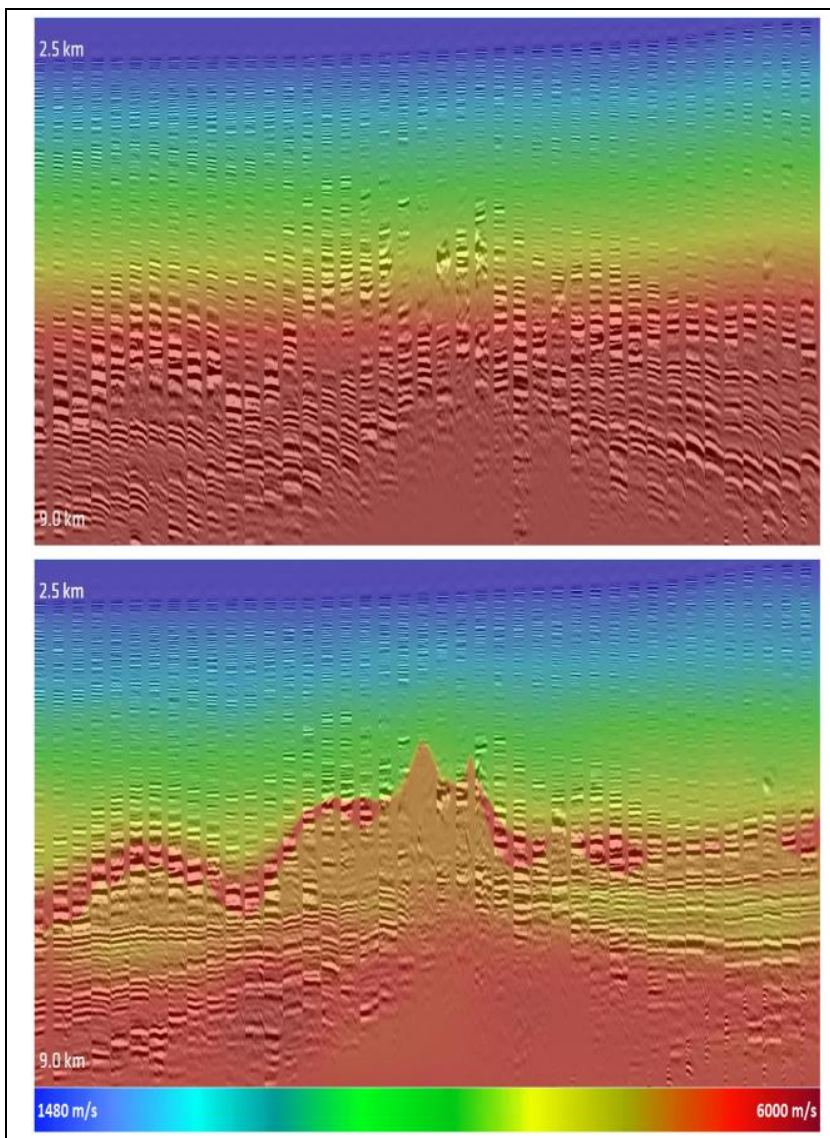

Figure 2. Common image gathers with $30^{0}$ mute before (top) and after (bottom) the automated analysis. Improved gather flatness is noticed throughout the section.

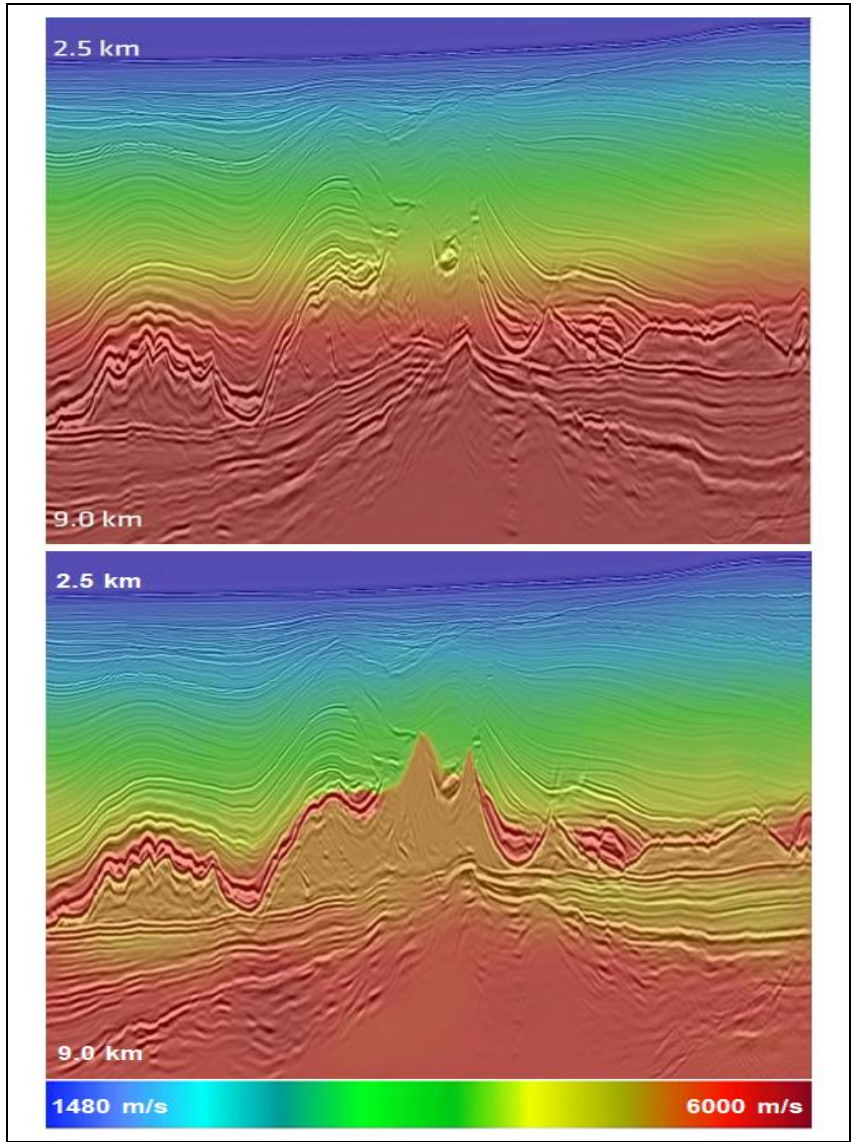

Figure 3. Migrated stack before (top) and after (bottom) the velocity update.

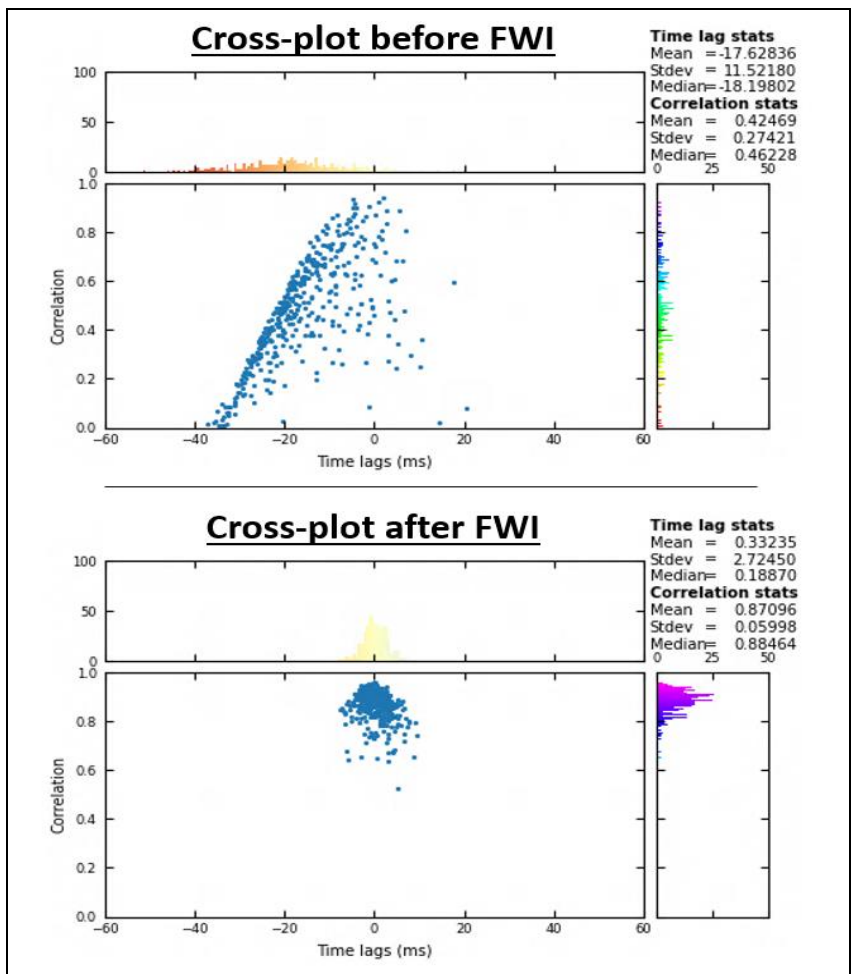

Figure 4. Cross plot of the crosscorrelation attributes before (top) and after (bottom) FWI. 


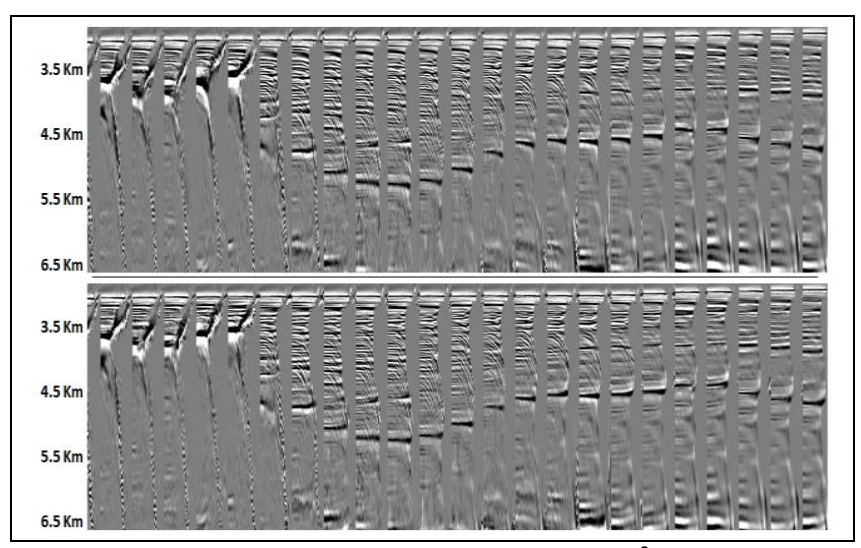

Figure 5. Common image gathers with $60^{\circ}$ mute before (top) and after (middle) FWI. In the bottom the FWI perturbation is overlaid on the stack.

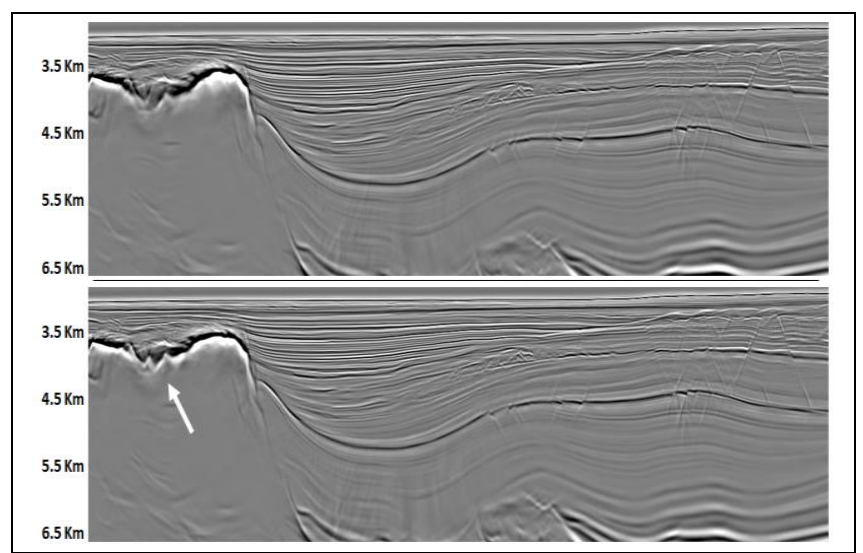

Figure 6. Migrated image before (top) and after (bottom) FWI update. Improvements in the salt geometry can be noticed after updating the velocity field (white arrow).

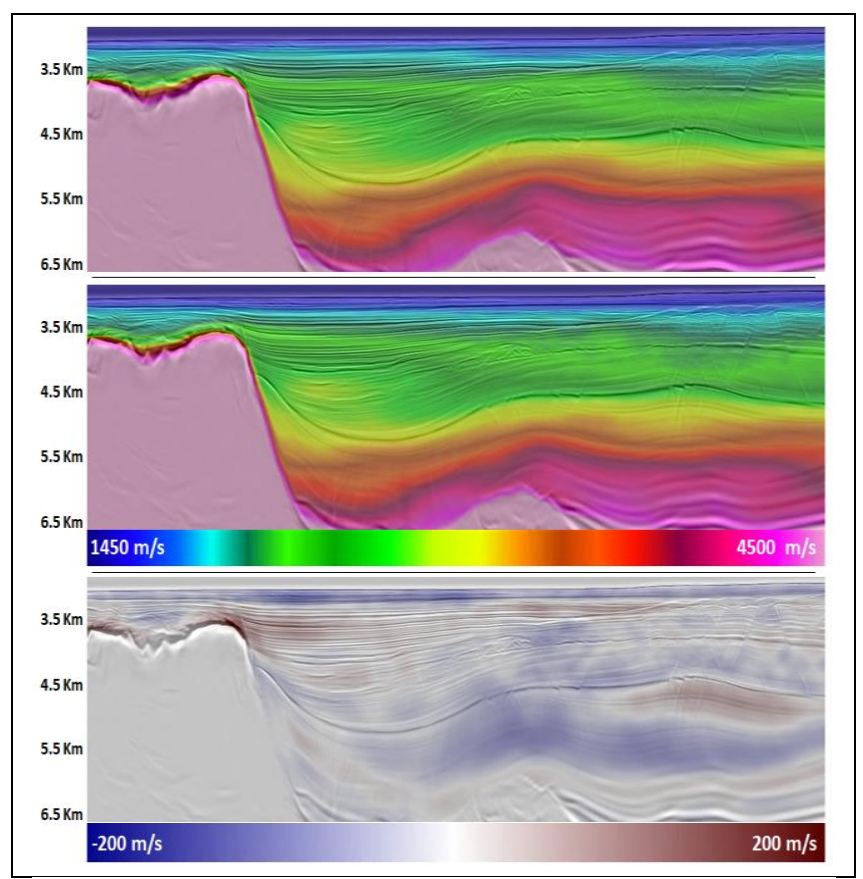

Figure 7. Velocity models before (top) and after FWI (middle). The perturbation (bottom) shows a smooth velocity update without cycle skipping. 